\title{
A Simple Model of Large Scale Organization in Evolution
}

\author{
S. C. Manrubia ${ }^{1}$ and M. Paczuski ${ }^{2,3}$ \\ ${ }^{1}$ CSRG, Departament de Física i Enginyeria Nuclear, UPC \\ c/ Sor Eulàlia d'Anzizu s/n Campus Nord, B4-B5. 08034 Barcelona, Spain \\ ${ }^{2}$ Department of Physics, Brookhaven National Laboratory, Upton NY 11973 \\ ${ }^{3}$ Department of Physics, University of Houston, Houston TX 77204-55063
}

\begin{abstract}
A mathematical model of interacting species filling ecological niches left by the extinction of others is introduced. Species organize themselves into genera of all sizes. The size of a genus on average grows linearly with its age, confirming a general relation between Age and Area proposed by Willis. The ecology exhibits punctuated equilibrium. Analytic and numerical results show that the probability distribution of genera sizes, genera lifetimes, and extinction event sizes are the same power law $P(x) \sim 1 / x^{2}$, consistent with paleontological data.
\end{abstract}

PACS number(s): 87.10.+e, 05.40.+j, 64.60.Lx

Typeset Using REVTEX 
Many years ago, Willis noted that genera could be composed of many species or of only one; he noted that similar regularities in the statistical properties of genera occured whether one is studying flowering plants or e.g. beetles. Attempting to formalize these observed regularites, he postulated a relation between the age and size of a genus, "Age and Area," which states that older genera on average include more species than younger ones [1]. With Yule he noted a power law relation for the number of genera with $s$ species, $P_{\text {gen }}(s) \sim s^{-\tau}$ with $\tau$ approximately 2 [1,2]. Recently, Burlando [3] observed scaling behavior across the taxonomic hierarchy also giving $\tau \simeq 2$ (see in addition Ref. 四). Similarly, the distribution of life times, $t$, of fossil genera [5] can be described by a power law $P_{\text {life }}(t) \sim 1 / t^{\tau_{t}}$ with $\tau_{t} \simeq 2$ [6]. When viewed at sufficiently large time scales, the pace of extinction itself is episodic with long periods of stasis interrupted by sudden bursts of mass extinction [8]. Punctuated equilibrium with scale-free extinctions has been attributed [9] to the self-organized critical [10] dynamics of strongly interacting species, without the need for catastrophic exogenous causes such as meteorites. The punctuated equilibrium process governing large scale evolution may be sufficiently robust or universal to be captured by an abstract, mathematical model. Bak and Sneppen [11] introduced a self-organized critical model of coevolving "species" where the least fit undergoes pseudo-extinction and affects the fitness of other species in the ecology, leading to extinction events of all sizes. However, their dimension-independent lifetime distribution $P_{\text {life }}(t) \sim 1 / t$ [6] is in disagreement with Raup's [0,6] paleontological data. More importantly, their model lacks any emergent taxonomic structure, which is an essential part of large scale organization in evolution.

Here we show that these three, seemingly unrelated distributions for extinction event sizes, genera sizes, and genera lifetimes, which characterize the large scale behavior of evolution, can be unified in terms of a simple mathematical process. We introduce an abstract model for large scale evolution and study it both analytically and numerically. In our model, surviving species can diversify into ecological niches left by previous extinction. This is implemented in terms of a Polya urn type of process. All species are subject to a general drift over time to lower viability which eventually leads to their extinction. This is consistent 
with the view that most changes in the ecology have a deleterious effect on currently existing species. In addition, due to interactions between species they experience changes in their viability, either favorable or unfavorable, arising from previous extinction of other species in the ecology. Species in our model organize themselves into genera of all sizes. The emergent genera obey a general Age and Area [1] relation which we find is linear $(s \sim t)$ in contrast to Yule's [2] conjecture $\ln s \sim t$. This large scale organization of species provides a simple mechanism which shapes all three probability distributions into a power law $P(x) \sim 1 / x^{2}$, where $x$ is the extinction event size, genera lifetime, or genera size. In all three cases the $1 / x^{2}$ behavior is consistent with previously reported paleontological data [5,6.9,3.3.1], indicating that our model may plausibly describe a universality class sufficiently broad to include real evolution. It can be tested further by directly comparing the age and size of extinct genera with our result that on average $s \sim t$.

Our model was inspired, in part, by considering a more complicated "connection" model introduced by Solé and Manrubia [12]. The advantage of our model is that it is extremely simple and robust. Its simplicity makes it easier to study large systems numerically; it is also analytically tractable. That such a simple model exists which describes a process giving large scale organization in evolution together with the above mentioned distributions is significant we think because it illustrates a potentially universal mechanism that would apply even beyond the context of biological evolution discussed here.

We begin by briefly describing the connection model. An $N \times N$ interaction matrix $W$ defines the interaction, either favorable or unfavorable, between $N$ objects that represent species. For a species $i$, the output elements $W_{i j}$ define its affect on the other species $j$, while its viability is the sum of its input elements $v(i)=\sum_{j} W_{j i}$. If $W_{j i}>W_{k i}$ then species $j$ has a more beneficial effect (and species $k$ has a more deleterious effect) on the ability of species $i$ to survive. If the viability $v(i)<0$, then species $i$ goes extinct, and the connection elements of the rows and columns for that extinct species are replaced with a copy of the corresponding elements of another surviving species. This copying in turn changes the viability of other species, and leads to a chain reaction of extinction events. The system is driven by slow 
random changes in the matrix $W$ which tend to lower the viability of the surviving species, and slowly differentiate copies from each other leading to speciation. They observed that the connection model exhibits extinction events of all sizes where the species that go extinct together tend to be recent copies.

In our one-dimensional model we assign to each of $N$ particles that represent species an integer viability $v(i)$. The dynamics consists of three steps as illustrated in Fig. 1: (1) species drift stochastically to lower viability; (2) species with viability below a threshold $v_{c}$ become extinct. The extinct species are each replaced with a "daughter" speciation of a surviving species. This is the Polya urn mechanism in our model; (3) Due to interactions between species the surviving species receive a change in their viability resulting from the extinction event. Specifically, at each time step the following operations are performed in parallel for all species $(i)$ : (1) with probability $1 / 2, v(i)=v(i)-1$; otherwise $v(i)$ is unchanged; $(2)$ for each $i$ such that $v(i)<v_{c}$ a surviving species $(j)$ with $v(j) \geq v_{c}$ is selected at random and $v(i)=v(j)$. This step represents a speciation event where one species branches into two. (3) all $N-s$ species that survived extinction receive a coherent influence $q(s)$, so that $v(j)=v(j)+q(s)$. After an extinction event of size $s, q(s)$ is chosen from the uniform distribution $-s \leq q(s) \leq s$. Thus, only large extinctions can cause large subsequent changes in the ecology. The form of $q(s)$ is elaborated on later. It is important to note that, unlike the connection model, our model's behavior is robust with respect to varying the parameter $v_{c}$, since the entire system is translationally invariant in viability.

Our model can be viewed as an example of transport in one dimension, where particles are conserved. In the steady state the smooth drift of species toward lower viability will be balanced by the intermittent replacement of extinct species with speciations of surviving ones, which by definition have higher viability. The average viability in the system $\bar{v}=$ $\frac{1}{N} \sum_{i} v(i)$ exhibits stick-slip behavior as shown in Fig. 2, similar to the behavior observed in the connection model [12].

Due to replacement of extinct species with speciations of surviving ones, the species tend to form groups with similar viability, which drift and diffuse together toward the extinction 
threshold. The state of the system may be characterized by $n(v, t)$, the number of species of viability $v$ at time $t$, where $\sum_{v} n(v, t)=N$. A snapshot of the system is shown in Fig. 3. At a microscopic scale, $n(v, t)$ is peaked with well defined bumps that give rise to the temporally intermittent sequence of extinctions as shown in the insert of Fig. 2.

We can identify all species within each bump as members of the same genus for the following reasons: Each viability bump is separated from the others by an empty interval where $n(v, t)=0$. Since these empty intervals cannot be filled by the replacement of extinct species with speciations of surviving ones, the dynamics tends to maintain the sharp separation between different bumps. Therefore, they are long-lived metastable entities. By making a histogram of the genera sizes, or area under each bump, observed in snapshots at spaced time intervals we find a power law for the number of species within each genus as shown in the insert of Fig. 3 with an exponent $\tau \simeq 2$. Also, the total number of genera in the system displays an intermittent pattern of diversification (increase) and contraction in time, qualitatively similar to real data [13].

The age of a newly created species following extinction is set to zero, and incremented by one unit at each step in the simulation. The age of a genus is the age of the oldest species in the corresponding bump. When a bump passes through the extinction threshold $v_{c}$, we measure its age $t$ and size $s$ (or area). The distribution of sizes of extinct genera is the same, within numerical accuracy, as the snapshot distribution described above. We numerically determined the relation between the age and size of extinct genera in a system of size $N=1000$ including $10^{7}$ time steps, and found a linear relation $t=m s$, with $m \simeq 0.6$. This numerical result indicates that emergent genera on average grow at constant rate irrespective of their size. The numerical result $\tau=2$, then implies $\tau_{t}=2$, in agreement with real data. Data collapse of the distribution of extinction event sizes for different system sizes also indicate a power law with exponent $\tau_{\text {ext }}=2$, as shown in Fig. 5. Note that at the beginning of the numerical simulation, with random initial conditions, there are only small extinctions and small genera. Thus the power law distributions observed in the steady state are "emergent"; they are consequences of the self-organized critical dynamics of our model. 
The first step in our model, drift to lower viability, takes into account slow random mutations of the matrix elements $W_{i j}$ in the connection model that tend to lower the viability of all species. This slow external driving, similar to that used in earthquake models [14, represents the cumulative effect on species of small changes in the environment, which we propose tend to make species less able to maintain their population over time. The second step represents true extinctions of species. The third step represents the effect of these extinctions on the surviving species. For more details see Ref. [15]. Our preliminary results indicate that the connection model also exhibits emergent genera with a broad size distribution, intermittent diversification, as well as an Age and Area relation.

We now discuss the analytic results for the transport model. In the stationary state, $P_{\text {ext }}(s)$ is the probability distribution to have an extinction event (avalanche) of size $s$ and $G(q)$ is the probability distribution to have an influence of size $q$. These distributions are self-consistently related via

$$
\begin{aligned}
G(q) & =\sum_{\text {all } s \geq q} \frac{P_{\text {ext }}(s)}{2 s+1} \\
P_{\text {ext }}(s) & =\sum_{\text {all } q} G(q) \delta\left(\sum_{v=0}^{q-1} \bar{n}(v), s\right),
\end{aligned}
$$

where $\bar{n}(v)$ is the time averaged viability profile in the steady state. The first equation is exact. The second assumes that the avalanche distribution comes from influences, $q$, on the time average viability profile, rather than the actual time dependent profile. The extinctions and influences are treated in terms of their full probability distributions, while the viability profile of species is treated only in terms of its average. This can be justified a posteriori in terms of a separation of scales argument similar to singular diffusion [16].

Next, we assume that the cumulant of $\bar{n}(v)$ is not singular around $v=0$, so that it has a Taylor series expansion. In the interval $1 \ll q \ll N$, where $N \rightarrow \infty, \int^{q} \bar{n}(v) d v=A q+\ldots$. Combining Eqs. $(1,2)$ with the Taylor series expansion gives

$$
\left.\frac{d G(q)}{d q}\right|_{q}=\frac{-1}{2 A q} G\left(\frac{q}{A}\right) \quad \text { for } \quad 1 \ll q \ll N .
$$

It is easy to show that Eq. (3) has a scaling solution $G(q) \sim q^{-\tau_{\text {ext }}}$ where $\ln \left(2 \tau_{\text {ext }}\right)=$ 
$\left(\tau_{\text {ext }}-1\right) \ln A$. Also the avalanche and influence distributions are asymptotically the same; $P_{\text {ext }}(q) \sim G(q)$.

The steady state equation for the time average profile is

$$
\bar{n}(v)=\frac{1}{2} \sum_{s=0}^{N-1} \sum_{q=-s}^{s} \frac{P_{e x t}(s)}{2 s+1}(\bar{n}(v+q)+\bar{n}(v+q+1))+\frac{1}{2}(\bar{n}(v)+\bar{n}(v+1)) \sum_{s=0}^{N-1} \frac{s P_{e x t}(s)}{N-s} .
$$

For large $N$, we try the solution $n(v)=n_{o} e^{-c v / N}$ and find to leading order in $N$

$$
1=\frac{1}{2} \sum_{s=0}^{N-1} \sum_{q=-s}^{s} \frac{P_{e x t}(s)}{2 s+1} e^{-c q / N}(2-c / N)+\sum_{s=0}^{N-1} \frac{s P_{e x t}(s)}{N-s} .
$$

Expanding for $q<<N$, the $q=0$ part of the first term on the right hand side gives $1-\frac{c}{2 N}$. The leading part cancels the number one on the left hand side of Eq. [0, and the negative remainder which comes from the drift must be cancelled by the remaining terms in the equation. Completing the expansion in $q$, only the even terms survive the symmetric sum over $q$. These terms are all positive as is the last term in Eq. 5. From Eq. (3) all of these positive terms scale $\sim N^{1-\tau_{e x t}}$. Only when $\tau_{\text {ext }}=2$ can the positive terms cancel the only negative term $\left(\frac{-c}{2 N}\right)$. In this case, a consistent solution exists for the exponential profile. Our numerical simulation results show that the average profile is indeed exponential with $c \simeq 6$ with $A \simeq 4$, both confirming $\tau_{e x t}=2$.

Note that our theory thus far has removed genera bumps by only treating the time average profile. The weak $\ln N$ divergence of both the average size of influences and average size of extinction events justifies our separation of scales assumption for large $N$. Since the shifts are small relative to $N$, only a finite number of genera on average pass the extinction threshold following an extinction. Then $\tau=\tau_{\text {ext }}=2$, in agreement with the numerical simulation result. Finally, previous interpretations of available data from the fossil record for extinction size distributions, genera (and higher order taxa) abundance distributions, and lifetime distribution of genera are consistent with our unified result in terms of a simple model that they are each decaying power laws with exponent 2.

This work was supported in part by the U. S. Department of Energy under Contract No. 
DE-AC02-76-CH00016 and DE-FE02-95ER40923 and by a grant of the Spanish Government DGYCIT 1995 PB94-1195. We thank P. Bak, M. Goldhaber, and R. V. Solé for interesting discussions about evolution. SCM acknowledges the hospitality of Brookhaven National Laboratory, where part of this work was done. 


\section{REFERENCES}

[1] J.C. Willis, Age and Area (Cambridge University Press, Cambridge, 1922).

[2] G.U. Yule, Phil. Trans. Roy. Soc. (London) CCXIII.-B(403), 21 (1924).

[3] B. Burlando, J. Theor. Biol. 146, 99 (1990); ibid 163, 161 (1993).

[4] C. Adami, C.T. Brown, and M.R. Haggerty, in Advances in Artifical Life, Proc. of the Third Europ. Conf. on Artificial Life, Lecture Notes in Artificial Intelligence 929, eds. F. Morán et. al. (Springer, New York, 1995).

[5] D.M. Raup, Extinction: Bad Genes or Bad Luck (Oxford University Press, Oxford, 1993); J.J. Sepkowski, Jr., Paleobiology 19, 43 (1991).

[6] K. Sneppen, P. Bak, H. Flyvbjerg, and M. H. Jensen, Proc. Nat. Acad. Sci. 92, 5209 (1995); P. Bak and M. Paczuski, ibid 92, 6689 (1995).

[7] The lifetime distribution in units of simulation time is different than the physically relevant time, which is argued to be exponential in fitness. This gives the universal behavior $1 / t$, even though the behavior in simulation time does depend on dimension.

[8] S. J. Gould and N. Eldredge, Paleobiology 3, 114 (1977); Nature 366, 223 (1993).

[9] S. A. Kauffman, Origins of Order: Self-Organization and Selection in Evolution (Oxford University Press, Oxford 1992).

[10] P. Bak, C. Tang, and K. Wiesenfeld, Phys. Rev. Lett. 59, 381 (1987); Phys. Rev. A. 38, 364 (1988).

[11] P. Bak and K. Sneppen, Phys. Rev. Lett. 71, 4083 (1993); M. Paczuski, S. Maslov, and P. Bak, Phys. Rev. E 53, 414 (1996).

[12] R. V. Solé, Complexity 1 (6) 512 (1996); R. V. Solé and S. C. Manrubia, Phys. Rev. E 54, 42 (1996). 
[13] D.H. Erwin, J.W. Valentine, and J.J. Sepkoski, Jr., Evolution 41 (6), 1177 (1987).

[14] Z. Olami, H.J.S. Feder, and K. Christensen, Phys. Rev. Lett. 68, 1244 (1992).

[15] Because they are close copies, the species which go extinct together in the connection model have similar output connections to any other species. Thus, the change in viability of any surviving species $j$ due to $s$ species going extinct involves the removal of $s$ correlated numbers contributing to the viability $v(j)$. This is why we use an influence distribution bounded by $s$, rather than e.g. $\sqrt{s}$. Using the $\sqrt{s}$ distribution gives $\tau_{\text {ext }}=1$. However, breaking the plus/minus symmetry of the influence distribution has no effect on the critical behavior as long as some influences are favorable, increasing viability. Note also that the value of $v_{c}$ in our model completely arbitrary, unlike the case for the connection model which is not critical unless $v_{c}=0$. Here we use $v_{c}=0$. In our model we use the same influence following extinction for all surviving species, rather than a different one for each species. In the connection model, not only are extinct species closely related, but we observe that the surviving species also form groups with similar matrix elements. Within each group or genus, all species will have similar connection to any other species, so that all species in the same genus get virtually the same effect in their viability from an extinction of another genus. In our model, we approximate the behavior of the connection model by assuming there is only one surviving genus group rather than many.

[16] J.M. Carlson and G. Swindle, Proc. Nat. Acad. Sci. 92, 6712 (1995). 


\section{FIGURES}

FIG. 1. Dynamics of the model. The horizontal axis is the viability and the blocks represent species. The dotted line is the extinction threshold. (0) Initial configuration. (1) Leftward stochastic drift. (2) Extinction and replacement. (3) Coherent influence to the survivors. Here the extinction had size $s=5$ and the influence of the extinction had value $q=-2$. The species that move at each step are shaded.

FIG. 2. The average viability as a function of time in a system of size $N=1000$ exhibiting stick-slip dynamics. The steep jumps, or slip events, are followed by slow relaxation to the threshold for extinction. The insert shows the temporal sequence of extinction event sizes over the same interval.

FIG. 3. A snapshot of the viability profile $n(v, t)$ in a system of size $N=400$. The pattern is intermittent with both small and large bumps. The insert shows the distribution of genera sizes

averaged over a total time interval of $10^{7}$ steps with a snapshot taken every 100 time steps for a system of size $N=1000$. The curve can be described as power law with a cutoff at the system size.

FIG. 4. Data collapse result. $P(s, N)$ is the probability to have an extinction event of size $s$ in a system of size $N$. The plateaus for different system sizes show that $P(s, N)=F(s / N) / s^{2}$ where $F(x)$ is simple scaling function which is constant for $x \ll 1$ and approaches zero as $x \rightarrow 1$. This agrees with our analytic result that $\tau_{\text {ext }}=2$. 


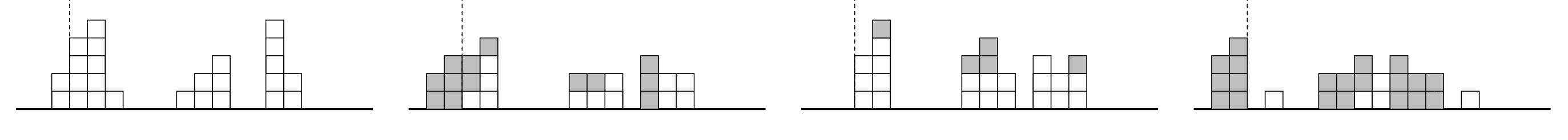




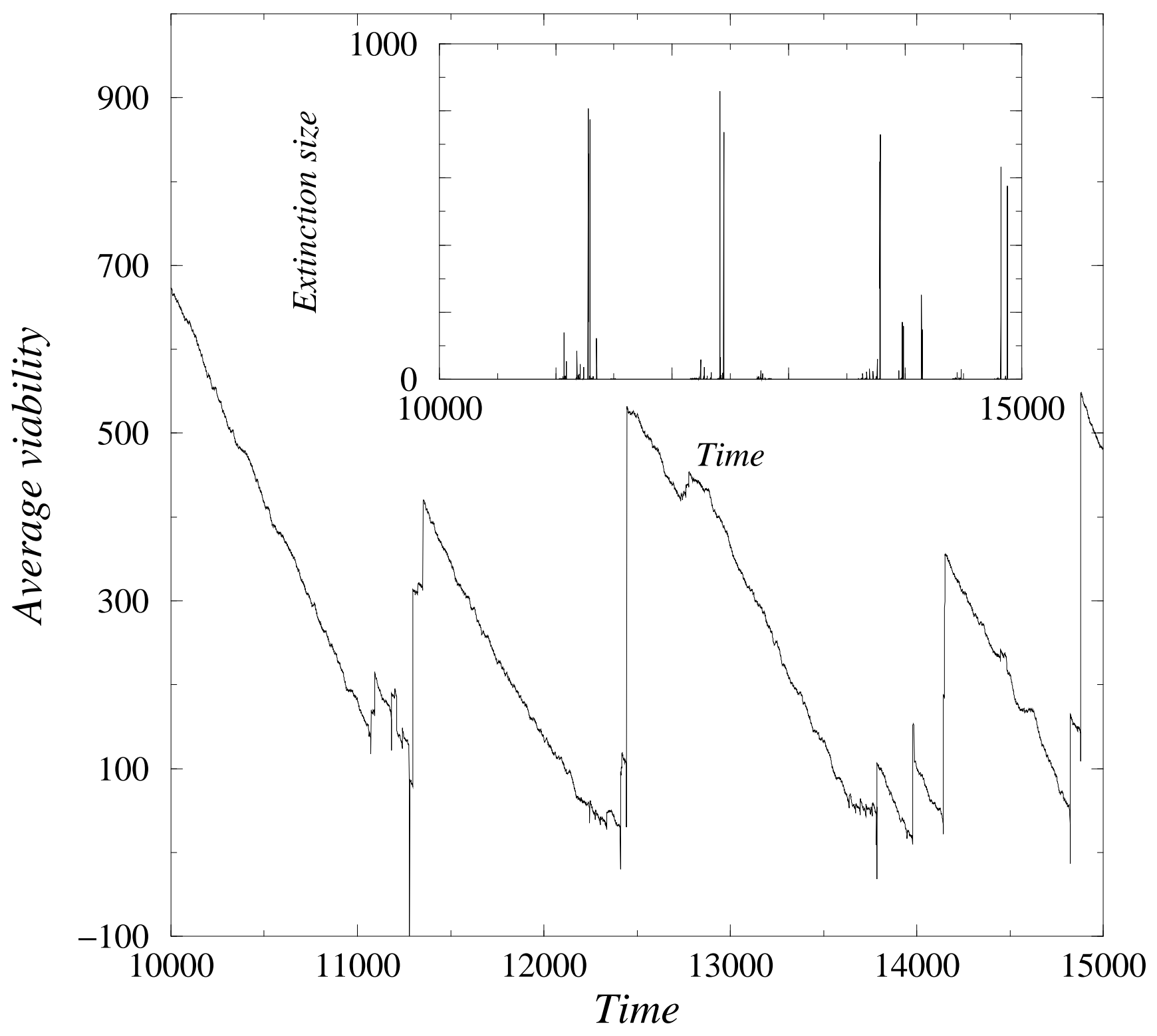




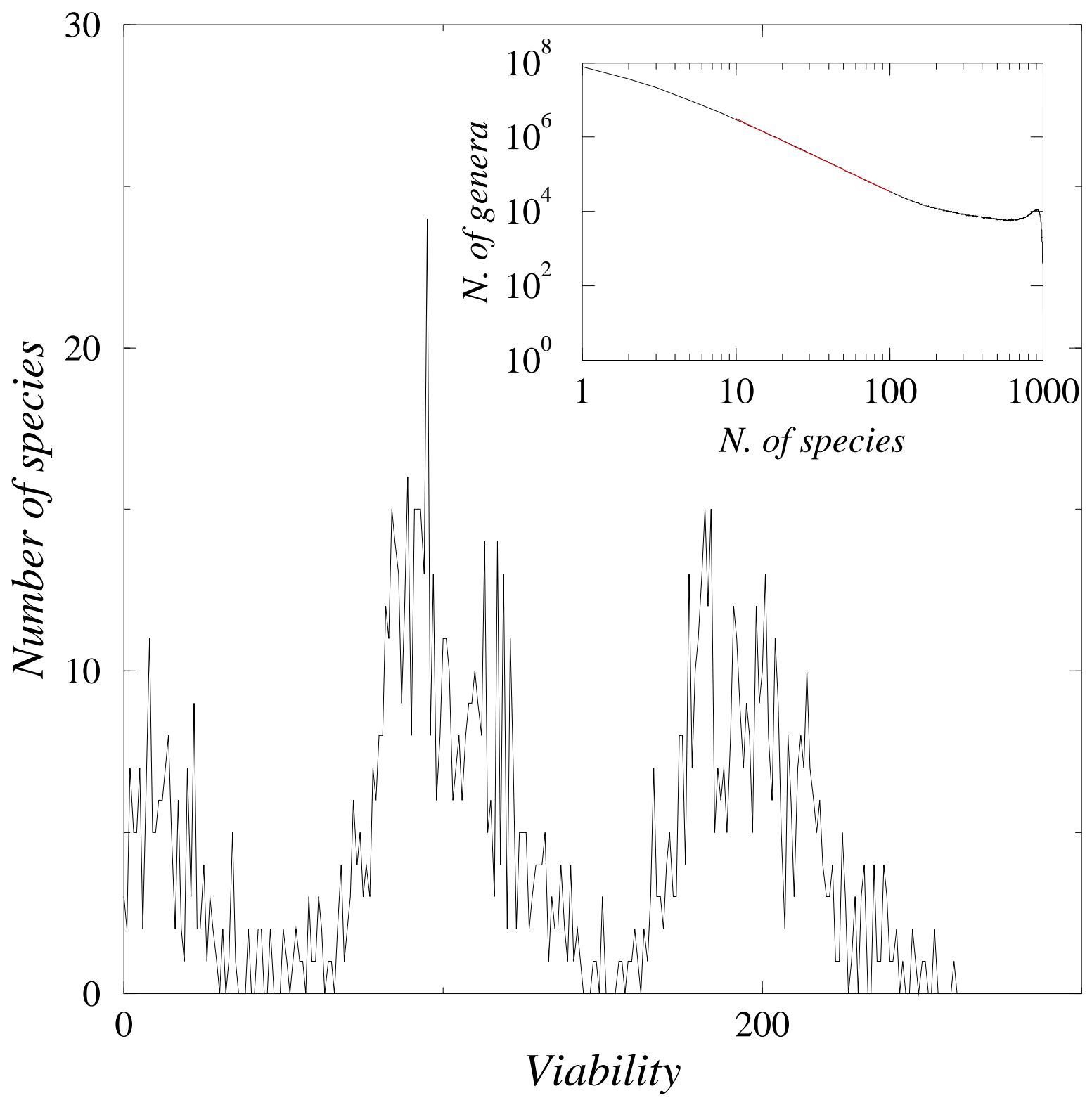




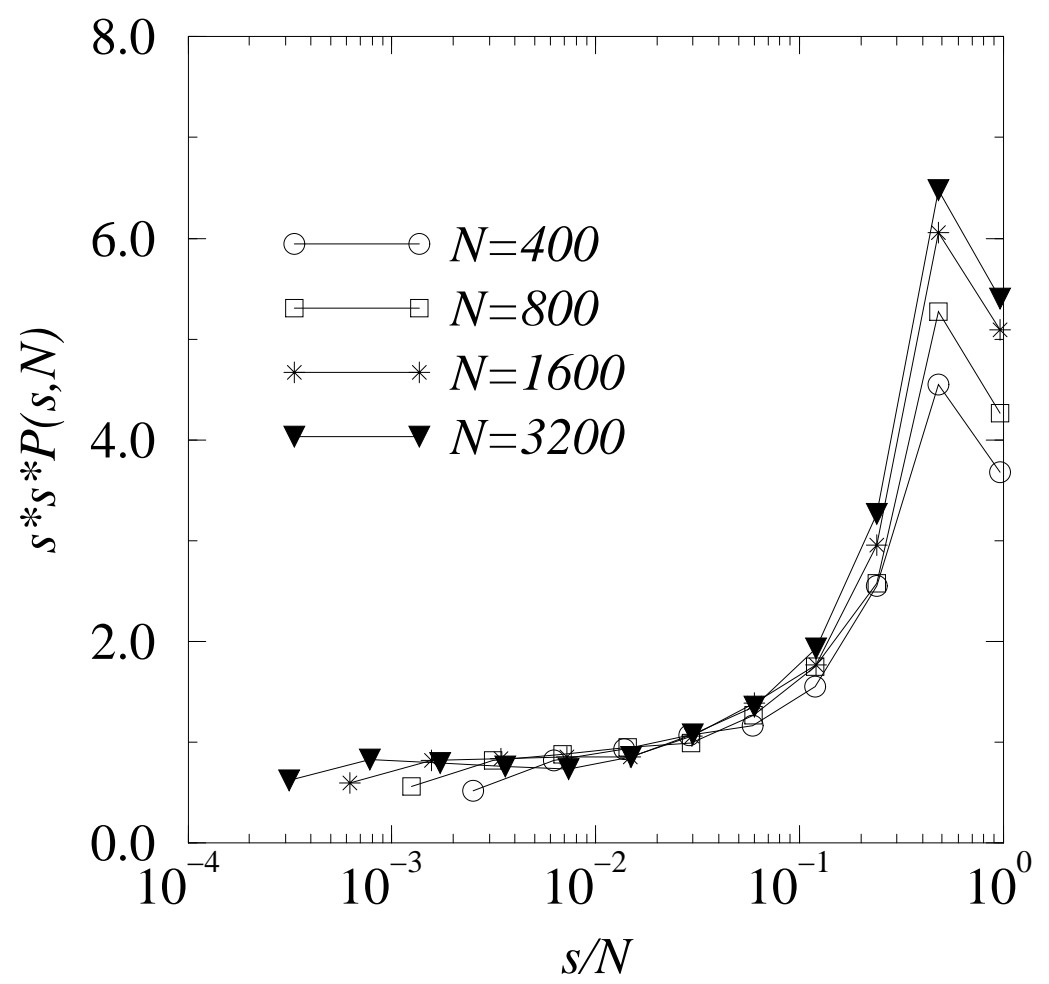

